\title{
Lecture intense en anglais LV2 : apport du multimédia
}

\section{Evelyn Perry}

\section{(2) OpenEdition}

\section{Journals}

Édition électronique

URL : http://journals.openedition.org/asp/1249

DOI : 10.4000/asp. 1249

ISBN : 978-2-8218-0394-7

ISSN : 2108-6354

Éditeur

Groupe d'étude et de recherche en anglais de spécialité

Édition imprimée

Date de publication : 1 novembre 2003

Pagination : 155-174

ISSN : 1246-8185

Référence électronique

Evelyn Perry, « Lecture intense en anglais LV2 : apport du multimédia », ASp [En ligne], 41-42 | 2003, mis en ligne le 29 avril 2010, consulté le 01 mai 2019. URL : http://journals.openedition.org/asp/1249 ; DOI : 10.4000/asp.1249

Ce document a été généré automatiquement le 1 mai 2019.

Tous droits réservés 


\title{
Lecture intense en anglais LV2 : apport du multimédia
}

\author{
Evelyn Perry
}

\section{Problématique}

1 Ayant expérimenté depuis plusieurs années des logiciels multimédia dans le cadre de l'enseignement universitaire de l'anglais L2, nous avons pu constater que les étudiants sont, en général, très heureux de se livrer à une séance informatique en langue vivante. Mais il convient de ne pas se laisser abuser par cet enthousiasme. Le caractère exceptionnel de nos séances multimédia, le fait que nous quittions notre salle de cours habituelle pour aller en salle informatique, contribuent sans doute à créer une atmosphère ludique. Ne serait-ce pas cet aspect-là qui plaît aux apprenants d'anglais L2 ? Si les expériences multimédia suscitent une réaction positive chez nos étudiants, sommes-nous pour autant en droit de dire que celles-ci sont plus utiles que l'enseignement traditionnel pour l'apprentissage d'une langue étrangère? Afin de tester l'efficacité du multimédia, nous avons comparé les performances réalisées par certains étudiants à partir d'une lecture intensive sur papier à celles d'autres apprenants confrontés à une présentation multimédia sur le même thème.

\section{Présentation de l'étude}

2 Suite à une première étude menée à l'université de Paris-Dauphine et dont les résultats ont été présentés en 1998 lors d'un colloque sur la lecture à l'université de Grenoble, nous avons voulu expérimenter les différences éventuelles entre une lecture classique sur papier et une nouvelle façon d'appréhender des informations par une approche multimédia. Nous sommes partie du principe que la nouvelle forme de «lecture», véhiculée par un environnement beaucoup plus riche en informations, serait avantageuse pour les deux grandes catégories d'apprenants, ceux qui intègrent plus facilement des 
données visuelles et ceux qui privilégient une assimilation auditive. En associant les deux approches, nous semble-t-il, il ne peut y avoir de perdants.

Dans le but de déterminer l'utilité relative d'une approche multimédia par rapport à une lecture classique, et ceci dans le domaine de la lecture intensive en L2, nous avons procédé dans un premier temps à une pré-étude, suivie d'une enquête plus approfondie. Les résultats de la première phase du projet nous ont permis de mieux orienter la seconde. Dans chaque phase les participants, tous étudiants du secteur LANSAD, ont expérimenté deux formes de lecture : une lecture traditionnelle des textes présentés sur un support papier et une présentation multimédia alliant image, voix et texte.

\section{Déroulement de l'expérience}

4 Au cours de l'expérience multimédia, abordée à l'aide d'un environnement multimédia, Help Yourself, élaboré par Alain Cazade à l'université Paris-Dauphine, les apprenants ont été invités à explorer des documents vidéo associés à des fichiers son, graphique et texte. Alors que la lecture traditionnelle était limitée aux textes imprimés, l'aspect textuel d'une "lecture " multimédia pouvait éventuellement se réduire à néant, le lecteur préférant regarder défiler les images et écouter le script lu par les présentateurs. Il était néanmoins possible à tout moment d'avoir accès au script, autrement dit à une lecture sans images, ni fond sonore. En effet, à l'intérieur de cet environnement, l'utilisateur avait plusieurs choix : visionner les images accompagnées d'une bande sonore, parcourir la transcription de la bande sans support auditif ou visuel, c'est-à-dire « lire » le script, ou regarder le script à l'écran tout en écoutant la bande sonore. Ces choix, volontairement limités par le concepteur de l'environnement Help Yourself, tiennent compte de la difficulté qu'éprouve l'utilisateur à rester pleinement attentif en phase de compréhension orale face à un texte écrit. La tendance, souvent évoquée, des données textuelles à compromettre l'impact du son joint à l'image, a notamment été abordée par Alain Cazade dans le document de synthèse pour obtenir une Habilitation à diriger des recherches (2000) et également étudiée et mise en perspective par Christine Vaillant dans une intervention proposée à l'occasion d'un séminaire au Louvre(1996). En ce qui concerne cette étude, les étudiants fonctionnant en mode multimédia ont eu comme consigne de commencer par la présentation comportant images et son.

5 Alors que pour l'étude liminaire nous n'avons disposé que de $24^{1}$ participants, tous étudiants en Magistère des sciences de gestion à l'université de Paris-Dauphine, nous avons pu élargir l'échantillon à deux promotions différentes de cette filière pour l'enquête approfondie, soit 56 sujets au total : 28 apprenants d'anglais L2 inscrits en première année, et 28 en troisième année. Pour les deux études nous avons procédé de la même manière ; chaque groupe de participants a été réparti en deux sous-groupes. L'un des deux a pratiqué une lecture classique, sur papier ; l'autre a découvert le même thème à l'aide d'un environnement multimédia. Cette procédure a nécessité deux séances pour chaque promotion, car le nombre de places disponibles dans notre centre de ressources en langues était limité à 15. Dans la deuxième phase du travail, les sujets ayant effectué une lecture traditionnelle lors de la première séance ont entamé la lecture enrichie en mode multimédia ; ceux qui avaient en revanche pratiqué une lecture dite multimédia du premier texte ont abordé un deuxième thème durant une phase de lecture sur papier. Le projet de recherche ainsi conçu a permis à chaque étudiant de réaliser deux production 
écrites, l'une fondée sur les documents parcourus en mode multimédia, l'autre prenant appui sur une lecture traditionnelle.

6 Les textes prévus pour le module multimédia, ainsi que pour la lecture sur papier, étaient les transcriptions, effectuées par nos soins, des bandes-vidéo réalisées pour l'émission télévisuelle CNN Newsroom. Le support du premier module, El Niño ou EN, portaitsur l'histoire de ce phénomène climatique; le second, Killer Viruses ou $K V$, était axé sur le thème des virus mortels provenant du continent africain (Ebola, Sida...).

7 Toutes les séances étaient chronométrées. Elles se décomposaient en deux parties: un premier temps de lecture de trente minutes, suivi d'une demi-heure de rédaction, pendant laquelle les apprenants fournissaient un résumé, rédigé en anglais L2, des documents qu'ils venaient d'étudier. Chaque participant a réalisé sa production écrite à l'ordinateur, grâce à un module éditeur de texte prévu dans l'environnement Help Yourself

Dans ce module, les étudiants sont censés rédiger un texte de cinq ou six paragraphes (3 000 caractères maximum), les paragraphes sont numérotés. Il suffit de cliquer sur l'un des chiffres pour faire démarrer la phase de rédaction. On peut également revenir sur une phrase ou expression déjà dactylographiée, afin d'effacer, par exemple, un mot mal orthographié ou de modifier le contenu d'un argument. Chaque intervention de l'utilisateur sur l'un des paragraphes est enregistrée et gardée en mémoire par l'ordinateur de manière à ce que toutes les versions du texte soient conservées dans leur intégralité, ceci à la différence d'un simple logiciel traitement de texte où seule la version définitive reste intacte. Par suite d'un problème technique, rectifié depuis, cette fonction n'a pas joué un grand rôle dans cette première étude, car la fonction étant activée par la touche retour de chariot, les participants l'ont trop souvent déclenchée sans le vouloir, pensant faire un simple retour à la ligne. Cependant, cette fonction constitue un outil précieux pour le chercheur s'intéressant aux processus intellectuels déployés lors de la production écrite, ainsi qu'à la capacité de l'apprenant d'identifier et corriger ses propres erreurs.

9 Afin d'analyser les productions écrites fournies, nous nous sommes proposé de mesurer l'efficacité de la lecture traditionnelle par rapport à l'expérience en mode multimédia en fonction de deux critères : le nombre d'informations perçues par les participants dans les documents étudiés et intégrées par la suite dans leurs essais et le nombre d'expressions utilisées dans les textes de base et que nous retrouvons dans les travaux rendus par les étudiants.

10 En ce qui concerne le premier critère, celui d'« information », nous avons opté pour une interprétation très large du terme. Il pourrait s'agir ici d'une donnée précise, telle qu'une date, le nom d'une personne ou d'un lieu, une statistique, etc. Mais nous avons également comptabilisé en tant qu'« information » les idées, principes ou arguments avancés dans les textes étudiés et repris ou reformulés par les étudiants.

11 Par le biais du deuxième critère, nous avons tenté de repérer l'efficacité relative de chaque mode de lecture en vue de l'assimilation et de la réutilisation des mots isolés ou d'expressions (collocations, idiomes) provenant des modules El Niño et Killer Viruses, chaque participant ayant reçu comme consigne de reproduire autant que possible le langage des documents parcourus. 


\section{Résultats de l'étude liminaire}

\subsection{Premier critère : information}

Les résultats de la première étude sont présentés pour chacun des deux textes dans les tableaux ci-dessous (Tableaux 1 à 3). Rappelons que pour cette partie de l'étude l'échantillon est composé de 21 étudiants, soit 12 en première année (Mag 1) et 10 en troisième année (Mag 3).

13 Chez les 12 participants inscrits en Magistère 1 (Tableau 1), nous ne trouvons qu'une différence insignifiante entre les travaux issus d'une lecture traditionnelle et les productions associées à un travail de lecture en mode multimédia. En effet, 78 informations ont été retenues par le groupe B (lecture traditionnelle) ; 71 par le groupe A (multimédia). La moyenne d'informations trouvées par le groupe A est de 11,8, alors que les participants du groupe B obtiennent une moyenne de 13.

Si nous examinons ensuite les performances des étudiants de troisième année (Tableau 1), nous constatons que, par rapport aux résultats attestés pour leurs camarades de première année, ces deux groupes de sujets semblent avoir été réellement influencés par le type de lecture pratiquée. Les 6 sujetsdu groupe multimédia ont reproduit un total de 39 informations: soit, une moyenne de 6,5. Les 4 participants du groupe B (papier), en revanche, ont enregistré 47 informations au total, avec une moyenne de 11,75. Mais peutêtre faut-il considérer ces résultats avec prudence, au vu de la taille de l'échantillon : 4 sujets seulement ayant pratiqué une lecture sur papier.

Tableau 1. Informations : El Niño (Mag 1 et 3, 1998)

\begin{tabular}{|l|c||c|c|}
\hline \multicolumn{1}{|c|}{ Sujet } & Lecture multimédia & Sujet & Lecture papier \\
\hline Mag 1 & Groupe A & Mag 1 & Groupe B \\
\hline$n^{\circ} 1$ & 10 & $n^{\circ} 6$ & 10 \\
\hline$n^{\circ} 2$ & 12 & $n^{\circ} 7$ & 5 \\
\hline$n^{\circ} 3$ & 18 & $n^{\circ} 8$ & 9 \\
\hline$n^{\circ} 4$ & 9 & $n^{\circ} 9$ & 17 \\
\hline$n^{\circ} 5$ & 6 & $n^{\circ} 10$ & 23 \\
\hline$n^{\circ} 12$ & 6 & $n^{\circ} 11$ & 14 \\
\hline$n^{\circ} 16$ & Groupe A & Mag 3 & Groupe B \\
\hline$n^{\circ} 15$ & $6 a g 3$ & $n^{\circ} 21$ & 10 \\
\hline \hline
\end{tabular}




\begin{tabular}{|c|c|c|c|}
\hline$n^{\circ} 17$ & 10 & $n^{\circ} 23$ & 16 \\
\hline$n^{\circ} 18$ & 2 & $n^{\circ} 24$ & 13 \\
\hline$n^{\circ} 19$ & 5 & & \\
\hline$n^{\circ} 20$ & 9 & & \\
\hline Total & 110 & Total & 125 \\
\hline Moyenne & 9,16 & Moy. & 12,5 \\
\hline
\end{tabular}

15 Toutefois l'analyse des résultats obtenus par les deux groupes d'étudiants confondus démontre que l'avantage de la lecture traditionnelle ne se maintient pas.

16 Quant aux productions écrites portant sur le document Killer Viruses (Tableaux 2 et 3), nous remarquons, en les analysant, que le nombre global d'informations provenant du document et réutilisées dans les travaux d'écriture est nettement inférieur à celui repéré pour l'ensemble des productions relatives au texte El Niño. Le nombre d'informations recueillies par les participants, tous groupes confondus, pour les séances El Niño s'élève à 235. Or nous ne trouvons que 91 informations au total dans les productions se rapportant au document Killer Viruses. En outre, si l'on se réfère aux colonnes 2 et 4 du Tableau 2, l'on voit bien que le nombre d'informations ayant trait au module Killer Viruses (info-textes) constitue souvent un très faible pourcentage de toutes les informations présentées par un participant (info-générales).

17 Au premier abord, cette disparité surprend, mais le déséquilibre est vraisemblablement dû à la formulation quelque peu différente du sujet associé à chaque module. La question posée sur le phénomène El Niño était présentée ainsi :

El Niño is primarily a media event, created by journalists hungry for a story. Discuss, giving concrete examples from this module or from other sources to support your views.

Nous avons proposé un choix de sujets pour le module Killer Viruses :

In your opinion, what could or should be done to prevent further outbreaks of killer diseases? Who should be put in charge of this programme?

ou

The perceived emergence of Aids, Ebola and other such killer diseases from deepest Africa is based less on fact than on unconscious cultural and racial prejudices against the "Dark Continent" (Africa). Discuss, giving reasons for your opinions.

Comme l'indiquent les phrases en italique, la tâche, telle que définie pour le module El Niño, invitait l'apprenant à reproduire autant que possible les idées et/ou les données factuelles du texte dont il venait de prendre connaissance. En revanche, les deux questions posées sur le module Killer Viruses incitaient l'étudiant à développer ses propres opinions sur les problèmes soulevés dans le document plutôt qu'à reformuler les faits ou les arguments des textes. 
Tableau 2. Informations : Killer Viruses (Mag 1, 1998)

\begin{tabular}{|c|c|c|c|}
\hline Sujet & $\begin{array}{c}\text { Lecture } \\
\text { Multimédia }\end{array}$ & Sujet & Lecture papier \\
\hline Groupe A & $\begin{array}{c}\text { info-textes/ } \\
\text { info-générales }\end{array}$ & Groupe B & $\begin{array}{l}\text { info-textes/ } \\
\text { info-générales }\end{array}$ \\
\hline$n^{\circ} 6$ & $0 / 18$ & $n^{\circ} 1$ & $3 / 17$ \\
\hline $\mathrm{n}^{\circ} 7$ & $1 / 12$ & $\mathrm{n}^{\circ} 2$ & $8 / 16$ \\
\hline$n^{\circ} 8$ & $1 / 18$ & $n^{\circ} 3$ & $7 / 19$ \\
\hline$n^{\circ} 9$ & $9 / 15$ & $n^{\circ} 4$ & $7 / 26$ \\
\hline$n^{\circ} 10$ & $1 / 32$ & $n^{\circ} 5$ & $3 / 13$ \\
\hline$n^{\circ} 11$ & $6 / 17$ & & \\
\hline Total & 18 & Total & 28 \\
\hline Moy. & 3 & Moy. & 5,6 \\
\hline $\begin{array}{l}\text { info-textes/ } \\
\text { info-gén. }\end{array}$ & $18,72 \%$ & $\begin{array}{l}\text { info-textes/ } \\
\text { info-gén. }\end{array}$ & $30,7 \%$ \\
\hline
\end{tabular}

19 L'analyse des résultats de cette phase de l'enquête, présentée dans les Tableaux 2 et 3 révèle qu'un plus grand nombre d'informations a été traité par les Magistère 1 ayant effectué une lecture préalable sur papier que chez leurs camarades ayant travaillé en mode multimédia. Ces derniers, au nombre de 6, ont exploité un total de 18 informations ayant trait au module. Les 5 étudiants ayant pratiqué une lecture sur papier en ont trouvé 28 , soit 5,6 en moyenne, par rapport à une moyenne de 3 attestée pour le groupe multimédia.

Les tendances décelées chez les sujets Magistère de troisième année (Tableau 3) semblent aller à l'encontre de celles analysées pour les Magistère de première année. Les sept étudiants de Magistère 3, groupe multimédia, ont obtienu une moyenne de 4,4 informations en relation avec le module, et ceci contre une moyenne de 3 pour les 5 sujets ayant effectué une lecture papier.

Tableau 3. Informations : Killer Viruses (Mag 3, 1998)

\begin{tabular}{|c|c|c|c|}
\hline Sujet & Lecture multimédia & Sujet & Lecture papier \\
\hline Groupe A & & Groupe B & \\
\hline apprenants & $12 / 21$ & & \\
\hline
\end{tabular}




\begin{tabular}{|l|c|l|l|}
\hline$n^{\circ} 26$ & $1 / 8$ & $n^{\circ} 32$ & $1 / 9$ \\
\hline$n^{\circ} 27$ & $1 / 14$ & $n^{\circ} 33$ & $1 / 16$ \\
\hline$n^{\circ} 28$ & $6 / 16$ & $n^{\circ} 34$ & $9 / 12$ \\
\hline$n^{\circ} 29$ & $0 / 12$ & $n^{\circ} 35$ & $0 / 9$ \\
\hline$n^{\circ} 30$ & $5 / 30$ & $n^{\circ} 36$ & $2 / 11$ \\
\hline$n^{\circ} 31$ & $4 / 11$ & $n^{\circ} 37$ & $5 / 13$ \\
\hline \hline Total & $\mathbf{2 9}$ & Total & $\mathbf{1 8}$ \\
\hline Moyenne & $\mathbf{4 , 1 4}$ & Moyenne & $\mathbf{2 , 6}$ \\
\hline info-textes / & & info-textes / & $\mathbf{2 4 , 8 3 \%}$ \\
\hline info-gén. & $\mathbf{2 3 , 9 0 \%}$ & Info-gén. & \\
\hline
\end{tabular}

21 L'écart entre la moyenne d'informations générales (16) obtenue en mode multimédia et celle, inférieure, de 11,67 atteinte en mode papier semble, lui aussi, attester d'une préférence pour le mode multimédia chez ces apprenants plus avancés. En revanche, la différence est infime en ce qui concerne les ratios obtenus à partir des informations tirées des textes étudiés en rapport avec le nombre global des informations présentées dans les essais : 23,90 \% pour la lecture multimédia ; $24,83 \%$ pour la lecture papier.

\subsection{Deuxième critère : mots clés}

Selon notre deuxième critère, celui de l'assimilation et de la réutilisation des mots ou expressions provenant des textes étudiés, la différence entre une lecture traditionnelle et une lecture enrichie multimédia paraît pencher légèrement en faveur de la lecture sur papier. Observons que pour les six sujets du groupe A (Tableau 4), nous trouvons un total de 31 termes ou expressions repris dans les documents (fichiers son et image, script) consultés en mode multimédia, soit une moyenne de 5,16. Les participants pratiquant une lecture sur papier ont fourni au total 43 mots clés ou expressions utilisés dans le texte de base, soit une moyenne de 7,16. Chez les Magistère 1, donc, la lecture classique semblerait avoir mieux marqué les étudiants en matière d'acquisition du vocabulaire.

Tableau 4. Mots clés : El Niño (Mag 1, 1998)

\begin{tabular}{|l|c||l|l|}
\hline Sujet & $\begin{array}{c}\text { Lecture } \\
\text { Multimédia }\end{array}$ & Sujet & Lecture papier \\
\hline Groupe A & & Groupe B & \\
\hline$n^{\circ} 1$ & 6 & $n^{\circ} 6$ & 8 \\
\hline$n^{\circ} 2$ & 5 & $n^{\circ} 7$ & 3 \\
\hline
\end{tabular}




\begin{tabular}{|l|c|l|l|c|}
\hline$n^{\circ} 3$ & 8 & $n^{\circ} 8$ & 10 \\
\hline$n^{\circ} 4$ & 8 & $n^{\circ} 9$ & 10 \\
\hline$n^{\circ} 5$ & 2 & $n^{\circ} 10$ & 8 \\
\hline$n^{\circ} 12$ & 2 & $n^{\circ} 11$ & 4 \\
\hline Total & 31 & Total & 43 \\
\hline Moyenne & 5,16 & Moyenne & 7,16 \\
\hline
\end{tabular}

Les étudiants de troisième année de Magistère ont fait preuve, eux aussi, d'une meilleure efficacité d'apprentissage lexical en anglais L2, lorsque leur travail s'est fait en mode lecture traditionnelle (Tableau 5). Dans l'expérience multimédia, les six apprenants du groupe A ont fourni 12 expressions tirées du texte $E l$ Niño, ce qui représente une moyenne de 2. Les quatre sujets du groupe B ayant travaillé sur papier ont reproduit 16 mots clés, soit une moyenne de 4. En comptabilisant les performances des quatre groupes issus des deux années, nous obtenons une moyenne de 5,9 pour les deux groupe B (papier) contre une moyenne de 3,6 pour les deux groupes multimédia.

Tableau 5. Mots clés : El Niño (Mag 3)

\begin{tabular}{|l|c|l|c|}
\hline \multicolumn{1}{|c|}{ Sujet } & Lecture multimédia & Sujet & Lecture papier \\
\hline Groupe A & & Groupe B & \\
\hline$n^{\circ} 15$ & 1 & $N^{\circ} 21$ & 5 \\
\hline$n^{\circ} 16$ & 0 & $N^{\circ} 22$ & 8 \\
\hline$n^{\circ} 17$ & 5 & $N^{\circ} 23$ & 0 \\
\hline$n^{\circ} 18$ & 1 & $N^{\circ} 24$ & 3 \\
\hline$n^{\circ} 19$ & 3 & & 16 \\
\hline$n^{\circ} 20$ & 2 & Total & \\
\hline Total & 12 & Moyenne & \\
\hline Moyenne & & & \\
\hline
\end{tabular}

24 En analysant les productions écrites portant sur le document Killer Viruses (Tableau 6), nous observons que chez les étudiants de première année les résultats obtenus sur le critère mots clés sont presque identiques pour les deux supports de lecture. La moyenne attestée pour le Groupe A multimédia est de 4 ; celle du Groupe B (lecture sur papier) est de 3,8. Cet équilibre ne se manifeste pas chez les étudiants de troisième année. Pour ces participants, la lecture traditionnelle semblerait avoir été plus efficace que la lecture 
multimédia, à en juger par les 34 expressions attestées (5,50 en moyenne) pour le Groupe $\mathrm{B}$, comparées à 17 items seulement (une moyenne de 2,83 ) répertoriés pour le groupe multimédia.

Tableau 6. Mots clés : Killer Viruses (Mag 1 et 3, 1998)

\begin{tabular}{|c|c|c|c|}
\hline Sujet & Lecture multimédia & Sujet & Lecture papier \\
\hline Mag 1 & Groupe A & Mag 1 & Groupe B \\
\hline$n^{\circ} 6$ & 2 & $n^{\circ} 1$ & 2 \\
\hline$n^{\circ} 7$ & 0 & $\mathrm{n}^{\circ} 2$ & 5 \\
\hline$n^{\circ} 8$ & 3 & $n^{\circ} 3$ & 4 \\
\hline$n^{\circ} 9$ & 13 & $n^{\circ} 4$ & 5 \\
\hline$n^{\circ} 10$ & 2 & $n^{\circ} 5$ & 3 \\
\hline$n^{\circ} 11$ & 4 & & \\
\hline Mag 3 & Groupe A & Mag 3 & Groupe B \\
\hline $\mathrm{n}^{\circ} 26$ & 1 & $n^{\circ} 32$ & 2 \\
\hline$n^{\circ} 27$ & 2 & $n^{\circ} 33$ & 7 \\
\hline$n^{\circ} 28$ & 3 & $n^{\circ} 34$ & 16 \\
\hline$n^{\circ} 29$ & 0 & $n^{\circ} 35$ & 2 \\
\hline$n^{\circ} 30$ & 8 & $n^{\circ} 36$ & 2 \\
\hline $\mathrm{n}^{\circ} 31$ & 3 & $n^{\circ} 37$ & 4 \\
\hline Total & 36 & Total & 53 \\
\hline Moyenne & 3,27 & Moyenne & 4,18 \\
\hline
\end{tabular}

\section{Deuxième phase de l'enquête}

Les résultats de la pré-étude, surtout en ce qui concerne l'assimilation des informations, nous ont permis de re-paramétrer la deuxième enquête. D'abord, les consignes citées cidessus ont été harmonisées. D'emblée il a été demandé à l'apprenant de rédiger un résumé du texte qu'il venait de lire, soit sur papier, soit à l'écran ; il lui a également été suggèré d'employer le plus possible de mots et d'expressions tirés du module. Suite à 
cette modification, les informations fournies dans des résumés de Killer Viruses collent parfaitement au texte, tout comme celles empruntées du module El Niño.

Les conditions nécessaires à une analyse statistique pertinente n'ayant pas été satisfaites en raison de la taille d'échantillon de la pré-étude, nous avons fait appel dans un deuxième temps à un nombre d'étudiants plus important afin de confirmer ou infirmer les tendances qui semblaient se profiler à l'issue de la phase préliminaire, c'est-à-dire une plus grande efficacité de la lecture traditionnelle.

\subsection{Résultat : informations}

En ce qui concerne les 28 participants de première année, nous avons trouvé une différence statistiquement significative en faveur d'une lecture multimédia pour les deux modules, $E N$ et $K V$ (voir Tableaux 7, 8, 11 et 12), alors qu'est apparue une différence significative en faveur du papier pour le module $E N$ chez les apprenants de troisième année (Tableaux 9, 10, 11 et 12). Chez ces derniers, cependant, il n'y a pas de différence significative entre les deux modes de lecture pour le module KV (Tableau 12). Si l'on considère les performances des deux promotions confondues, il n'apparaît pas non plus de différence significative entre les deux types de lecture pour le module $E N$, ni pour $K V$ (Tableau 12).

\subsection{Résultats : mots clés}

Les étudiants inscrits en première année de Magistère de gestion (Mag 1) ont réalisé de meilleures performances en termes de mot-clés réutilisés dans leur production écrite après une lecture du module $E l$ Niño effectuée en mode multimédia ; pour le module Killer Viruses, nous n'avons pas trouvé de différence significative pour ces apprenants (voir Tableau 12).

Tableau 7. Mag 1, Groupe A - résultats individuels 1999

\begin{tabular}{|l|c|c||c|c|}
\hline Sujet & $\begin{array}{c}\text { Infos } \\
E N \\
M m\end{array}$ & $\begin{array}{c}\text { Mots clés } \\
\text { Mm }\end{array}$ & $\begin{array}{c}\text { Infos } \\
\text { KV } \\
\text { Papier }\end{array}$ & $\begin{array}{c}\text { Mots clés } \\
\text { KV } \\
\text { Papier }\end{array}$ \\
\hline$n^{\circ} 42$ & 35 & 17 & 24 & 8 \\
\hline$n^{\circ} 43$ & 30 & 12 & 20 & 15 \\
\hline$n^{\circ} 44$ & 29 & 8 & 24 & 15 \\
\hline$n^{\circ} 45$ & 31 & 8 & 30 & 12 \\
\hline$n^{\circ} 46$ & 17 & 11 & 26 & 9 \\
\hline$n^{\circ} 47$ & 32 & 9 & 32 & 15 \\
\hline$n^{\circ} 48$ & 32 & 5 & 22 & 8 \\
\hline
\end{tabular}




\begin{tabular}{|l|c|c|c|c|c|}
\hline$n^{\circ} 49$ & 20 & 3 & 23 & 5 \\
\hline$n^{\circ} 50$ & 24 & 8 & 24 & 6 \\
\hline$n^{\circ} 51$ & 29 & 13 & 28 & 14 \\
\hline$n^{\circ} 52$ & 25 & 8 & 21 & 12 \\
\hline$n^{\circ} 53$ & 29 & 14 & 32 & 26 \\
\hline$n^{\circ} 54$ & 24 & 11 & 33 & 13 \\
\hline Total & $\mathbf{3 5 7}$ & $\mathbf{1 2 7}$ & $\mathbf{3 3 9}$ & $\mathbf{1 5 8}$ \\
\hline Moyenne & $\mathbf{2 7 , 4 6}$ & $\mathbf{9 , 7 7}$ & $\mathbf{2 6 , 0 7}$ & $\mathbf{1 2 , 1 5}$ \\
\hline
\end{tabular}

Tableau 8. Mag 1, Groupe B - résultats individuels 1999

\begin{tabular}{|l|c|c|c|c|}
\hline Sujet & $\begin{array}{c}\text { Infos } \\
E N\end{array}$ & $\begin{array}{c}\text { Mots clés } \\
E N \\
\text { Papier }\end{array}$ & $\begin{array}{c}\text { Papier } \\
\text { KV } \\
\text { Mm }\end{array}$ & $\begin{array}{c}\text { Mots clés } \\
\text { KV } \\
\text { Mm }\end{array}$ \\
\hline$n^{\circ} 27$ & 29 & 6 & 18 & 10 \\
\hline$n^{\circ} 28$ & 31 & 9 & 21 & 12 \\
\hline$n^{\circ} 29$ & 23 & 2 & 22 & 11 \\
\hline$n^{\circ} 30$ & 28 & 6 & 21 & 10 \\
\hline$n^{\circ} 31$ & 25 & 8 & 23 & 10 \\
\hline$n^{\circ} 32$ & 19 & 4 & 21 & 2 \\
\hline$n^{\circ} 34$ & 21 & 10 & 18 & 12 \\
\hline$n^{\circ} 35$ & 19 & 7 & 23 & 18 \\
\hline$n^{\circ} 36$ & 21 & 4 & 18 & 7 \\
\hline$n^{\circ} 37$ & 29 & 15 & 20 & 6 \\
\hline$n^{\circ} 38$ & 15 & 6 & 23 & 17 \\
\hline$N^{\circ} 41$ & 25 & 10 & 28 & 10 \\
\hline
\end{tabular}




\begin{tabular}{|l|c|c|c|c|c|}
\hline Total & 301 & 93 & 276 & 136 \\
\hline \hline Moyenne & 23,15 & 7,15 & 21,23 & 10,46 \\
\hline
\end{tabular}

Pour la promotion sortante, les Mag 3, les performances fondées sur une lecture papier ont donné des résultats positifs pour la réutilisation de mots clés mais seulement en rapport avec le texte Killer Viruses; aucune différence significative dans la rubrique mots clés n'a été relevée pour le module $E l$ Niño. Au bout du compte, lorsque nous considérons les performances des 56 étudiants des deux promotions comme un seul échantillon, il n'y a pas de différence significative en termes d'acquisition du vocabulaire entre les deux modes de lecture, ni pour un texte, ni pour l'autre.

Autrement dit, les étudiants de Magistère de gestion première année ont mieux fonctionné, trois fois sur quatre, en mode multimédia; leurs condisciples de troisième année semblent avoir été moins attirés, ou moins motivés, par le multimédia, et ont obtenu des résultats plus nuancés. Les Mag 3 ont atteint leurs meilleures performances deux fois en mode traditionnel (El Niño, critère : informations, Killer Viruses, critère : mots clés) ; pour les deux autres créneaux, (El Niño, critère : mots clés, Killer Viruses, critère : informations) ils ne semblent pas plus stimulés par le papier que par le multimédia. Ces résultats ne coïncident pas avec ceux obtenus par Alain Cazade dans une étude menée à la même époque (1998) et présentée dans ce recueil d'articles. Alain Cazade a trouvé une meilleur maitrise de l'outil multimédia chez les apprenants plus avancés participant à son étude (c'est-à-dire des étudiants de troisième année de Magistère de gestion) que chez les apprenants d'un niveau plus faible. Nous y reviendrons.

Tableau 9. Mag 3, Groupe A - résultats individuels 1999

\begin{tabular}{|l|c|c||c|c|}
\hline Sujet & $\begin{array}{c}\text { Infos } \\
\text { KV } \\
\text { papier }\end{array}$ & $\begin{array}{c}\text { Mots clés } \\
\text { KV } \\
\text { Papier }\end{array}$ & $\begin{array}{c}\text { Infos } \\
\text { EN }\end{array}$ & $\begin{array}{c}\text { mots clés } \\
\text { EN } \\
\text { Mm }\end{array}$ \\
\hline$n^{\circ} 1$ & 21 & 9 & 20 & 8 \\
\hline$n^{\circ} 2$ & 23 & 10 & 15 & 7 \\
\hline$n^{\circ} 3$ & 18 & 9 & 18 & 7 \\
\hline$n^{\circ} 4$ & 31 & 8 & 26 & 7 \\
\hline$n^{\circ} 5$ & 24 & 11 & 16 & 7 \\
\hline$n^{\circ} 6$ & 14 & 23 & 18 & 17 \\
\hline \hline$n^{\circ} 7$ & 21 & 11 & 15 & 4 \\
\hline$n^{\circ} 8$ & 20 & 13 & 20 & 8 \\
\hline
\end{tabular}




\begin{tabular}{|l|c|c|c|c|}
\hline$n^{\circ} 9$ & 18 & 8 & 21 & 6 \\
\hline$n^{\circ} 10$ & 24 & 8 & 15 & 4 \\
\hline$n^{\circ} 11$ & 17 & 5 & 19 & 7 \\
\hline$n^{\circ} 12$ & 26 & 17 & 19 & 3 \\
\hline$n^{\circ} 13$ & 20 & 9 & 24 & 9 \\
\hline Total & 277 & 146 & $\mathbf{2 4 6}$ & 94 \\
\hline Moyenne & $\mathbf{2 1 , 3 1}$ & $\mathbf{1 1 , 2 3}$ & $\mathbf{1 8 , 9 2}$ & $\mathbf{7 , 2 3}$ \\
\hline
\end{tabular}

Tableau 10. Mag 3, Groupe B - résultats individuels 1999

\begin{tabular}{|l|c|c||c|c|}
\hline Sujet & $\begin{array}{c}\text { Infos } \\
\text { KV } \\
\text { m-m }\end{array}$ & $\begin{array}{c}\text { Mots clés } \\
\text { KV } \\
\text { m-m }\end{array}$ & $\begin{array}{c}\text { Infos } \\
\text { EN } \\
\text { Papier }\end{array}$ & $\begin{array}{c}\text { Mots clés } \\
\text { EN } \\
\text { Papier }\end{array}$ \\
\hline$n^{\circ} 14$ & 24 & 9 & 27 & 11 \\
\hline$n^{\circ} 15$ & 22 & 7 & 25 & 5 \\
\hline$n^{\circ} 16$ & 25 & 6 & 24 & 7 \\
\hline$n^{\circ} 17$ & 16 & 3 & 23 & 10 \\
\hline$n^{\circ} 18$ & 30 & 10 & 32 & 8 \\
\hline$n^{\circ} 19$ & 20 & 11 & 23 & 8 \\
\hline$n^{\circ} 20$ & 13 & 6 & 28 & 12 \\
\hline$n^{\circ} 21$ & 27 & 14 & 22 & 6 \\
\hline$n^{\circ} 22$ & 26 & 4 & 26 & 2 \\
\hline$n^{\circ} 23$ & 19 & 8 & 15 & 3 \\
\hline$n^{\circ} 24$ & 20 & 4 & 23 & 3 \\
\hline$n^{\circ} 25$ & 27 & 12 & 24 & 5 \\
\hline Total $^{\circ} 26$ & 293 & 101 & 312 & 91 \\
\hline 2,53 & 7,76 & 24,00 & 7100 \\
\hline
\end{tabular}


Tableau 11. Informations et mot-clés : moyennes par groupe 1999

\begin{tabular}{|c|c|c|}
\hline & MAG 1 & Groupe A \\
\hline & Informations & Mots clés \\
\hline M-M & 27,5 (EN) & $9,8 \quad(\mathrm{EN})$ \\
\hline \multirow[t]{3}{*}{ Papier } & $26,1 \quad(\mathrm{KV})$ & $12,2 \quad(\mathrm{KV})$ \\
\hline & MAG 1 & Groupe B \\
\hline & Informations & Mots clés \\
\hline M-M & $21,2 \quad$ (KV) & $10,5 \quad(\mathrm{KV})$ \\
\hline \multirow[t]{3}{*}{ Papier } & 23,2 (EN) & $7,15 \quad(\mathrm{EN})$ \\
\hline & MAG 3 & Groupe A \\
\hline & Informations & Mots clés \\
\hline $\mathrm{M}-\mathrm{M}$ & $18,9 \quad$ (EN) & $7,2 \quad$ (EN) \\
\hline \multirow[t]{3}{*}{ Papier } & $21,3 \quad(\mathrm{KV})$ & $10,9 \quad(\mathrm{KV})$ \\
\hline & MAG 3 & Groupe B \\
\hline & Informations & Mots clés \\
\hline M-M & $22,5 \quad$ (KV) & $7,7 \quad(\mathrm{KV})$ \\
\hline Papier & $24,0 \quad(\mathrm{EN})$ & $7,0 \quad$ (EN) \\
\hline
\end{tabular}

Tableau 12. Mag 1 et Mag 3 confondus 1999

\begin{tabular}{|l||c|}
\hline & $\begin{array}{r}\text { Résultats significatifs } \\
\text { En faveur de : }\end{array}$ \\
\hline Mag 1 & \\
\hline Infos - kv & Papier \\
\hline Infos - en & M-M \\
\hline Mots - kv & Néant \\
\hline Mots - en & M-M \\
\hline
\end{tabular}




\begin{tabular}{|l|c|}
\hline Mag 3 & \\
\hline Infos - kv & Néant \\
\hline Infos - en & Papier \\
\hline Mots - kv & Papier \\
\hline Mots - en & Néant \\
\hline Mag 1 + Mag 3 \\
\hline Infos - kv & Néant \\
\hline Infos - en & Néant \\
\hline Mots - kv & Papier \\
\hline Mots - en & Néant \\
\hline
\end{tabular}

\subsection{Discussion}

En début d'enquête, nous postulions un rendement plus efficace pour les apprenants LANSAD travaillant en mode multimédia que pour leurs camarades effectuant une lecture traditionnelle sur papier. Les résultats de notre premier sondage, ou pré-étude, ont été quelques peu surprenants car l'avantage supposé du multimédia n'a pas été validé. $\mathrm{Au}$ contraire, les participants confrontés à une lecture classique sur papier ont obtenu des résultats plus satisfaisants que ceux qui ont eu accès aux modules multimédia. Sachant que le nombre trop faible de participants pouvait mettre en question la fiabilité des analyses statistiques appliquées à cette première phase de notre enquête, nous nous sommes proposé de refaire l'enquête avec un échantillon plus important.

En effet, les résultats issus de la deuxième phase de l'enquête diffèrent sensiblement de ceux de la première. Le schéma qui se dessine au vu de ces derniers résultats est celui de deux groupes d'apprenants distincts. D'un côté sont rangés les étudiants Bac + 3 (Mag 1), de l'autre les Bac + 5 (Mag 3). Les Mag 1, plus jeunes de deux ans seulement, semblent plus stimulés par l'environnement multimédia que leurs aînés. Pour une tâche sur deux, leurs performances s'améliorent face à un module multimédia, comme l'indiquent les moyennes cités dans les Tableaux 7 et 8, (voir aussi les Tableaux 11 et 12 ). La mémorisation à court terme et la réutilisation des informations présentées dans les textes $E N$ profitent de ce mode de lecture; l'assimilation des informations du module $K V$, en revanche, semble être favorisée par le mode papier.

En ce qui concerne les mots clés et leur réutilisation dans les productions écrites, le langage du texte apparaît de nouveau mieux intégré suite à une séance multimédia pour un module sur deux. Les résultats concernant le vocabulaire du module El Niño ont manifestement été favorisés par une lecture multimédia, alors que cette préférence pour le travail multimédia n'est pas avéréede manière significative pour le réemploi des expressions du module Killer Viruses. Une remarque peut être faite : les Mag 1 ont obtenu 
de meilleurs résultats en mode multimédia uniquement sur les textes $E l$ Niño. Le module Killer Viruses a pourtant résisté à cette préférence. Pour la catégorie "mots» de ce module, aucune différence significative entre les deux types de lecture n'a été relevée ; en ce qui concerne le critère "information », une différence statistiquement significative a été attestée mais en faveur de la lecture classique.

Les étudiants de troisième année de Magistère, en revanche, ne semblent pas avoir partagé l'enthousiasme des Magistère première année pour le multimédia, si nous en jugeons par leurs performances attestées ici. Celles-ci ne permettent pas de trancher clairement en faveur de l'un ou l'autre des deux modes de fonctionnement: lecture traditionnelle, lecture multimédia. Les résultats provenant de ce groupe ont plutôt penché en faveur d'une lecture traditionnelle. Par exemple, leur travail a été meilleur en mode papier, en ce qui concerne la catégorie «mot-clés », de Killer Viruses ainsi que pour la rubrique «informations » du module $E l$ Niño. Aucune préférence ne s'est affichée pour les «mot-clés » de Killer Viruses, ni pour les «informations » de El Niño. L'absence des résultats statistiquement significatifs caractérise aussi les deux groupes vus comme un ensemble, à l'exception de la rubrique "mot-clés» de Killer Viruses, où la lecture traditionnelle l'emporte pour les deux groupes confondus, tout comme pour les Mag 3. Le tableau récapitulatif, Tableau 12, regroupant les performances statistiquement différentes, met en relief ces deux grandes tendances chez les étudiants des deux promotions.

Comment expliquer cette disparité ? Pourquoi les Mag 3 auraient-ils été plus performants sortant d'une séance de lecture classique que d'une expérience multimédia? Doit-on interpréter ces résultats de manière négative ou positive ? En d'autres termes, les Mag 3 se désintéressaient-ils du multimédia ou éprouvaient-ils un réel attachement à la lecture traditionnelle? Une préférence pour un support plus sobre, moins saturé d'informations?

Il est peu probable que l'on puisse expliquer la différence entre les Mag1 et les Mag 3 par un goût pour le multimédia chez les plus jeunes contre une peur éventuelle de ce mode de fonctionnement chez les Mag 3, comme celapourrait être le cas avec une population plus âgée et peu habituée à un travail informatisé. La différence d'âge n'est que de deux ans entre les deux promotions et la différence de formation est quasi-inexistante. L'étude, menée en 1998-1999 ne date pas d'une époque complètement révolue avant l'incursion de l'ordinateur dans nos activités quotidiennes les plus banales.

En outre, cette population estudiantine plutôt privilégiée a toujours eu accès aux ordinateurs, depuis la première année de leurs études universitaires, sinon au lycée. La filière Magistère des sciences de gestion réserve à ses élèves une salle informatique bien équipée, qu'ils fréquentent pour préparer leurs travaux dans bon nombre de disciplines. Enfin, les cours de langue sont souvent complétés par des séances multimédia pendant lesquelles ces étudiants apprennent, dès la première année de la formation, à manier l'environnement Help Yourself pratiqué lors de cette étude. En effet, ils passent leurs examens d'anglais dans le même Centre de Ressources en Langues utilisés pour la présente étude. Les étudiants de troisième année sont donc a priori des individus qui ne doivent éprouver aucune réticence devant un ordinateur.

Si le critère de familiarité et d'aisance devant l'écran n'a pas pu jouer, ne serait-ce pas peut-être tout le contraire? Que les sujets en dernière année de Magistère se lassent de l'informatique, et par association, du multimédia? Ce qui a été amusant et perçu comme novateur au départ, c'est-à-dire comme nous l'avons souligné en début d'article, l'aspect 
ludique associé à des séances exceptionnelles proposées dans les laboratoires de langues informatisés, peut devenir par la suite banal. Pour prolonger cette interprétation, on peut avancer que les étudiants en fin de première année n'ont pas encore eu le temps de faire ce chemin. Il serait intéressant de répéter l'étude aujourd'hui, cinq ans plus tard, à une époque où tout laisse supposer que la magie de l'informatique a en bonne partie disparu avec la présence massive de l'Internet dans la vie des étudiants.

D'instinct, nous proposerions plutôt comme hypothèse que les Mag 3, en fin de cursus purement académique, se sont moins intéressés à toute tâche scolaire que les Mag 1, moins blasés par rapport à leurs études. Ayant passé le semestre précédent dans une université étrangère, les Mag 3 rentrent pour passer onze semaines seulement à l'université avant de quitter définitivement les salles de cours. En mars, vers la fin de cette dernière période de scolarité, à l'époque où s'est déroulée l'enquête, ils n'ont chaque année qu'une seule préoccupation majeure : trouver un stage en entreprise. Pendant leur séjour à l'étranger, expérience qui se passe pour la plupart d'entre eux dans des pays anglo-saxons, ils ont tous, certes, amélioré leur niveau d'anglais. Plus important encore, ils sont rentrés plus mûrs, impatients de sortir du berceau de l'université et d'entrer dans la vie active.

Pour toutes ces raisons, les Mag 3 constituent une population différente, intellectuellement ou psychologiquement moins disponible que les Mag 1. Ces derniers sont toujours soucieux du bon déroulement de leur parcours universitaire, et de ce fait, plus friands de toute activité susceptible de les aider. Ils se prêtent volontiers au jeu de l'expérience, même si - ou parce que - l'enseignant a pris soin d'annoncer que l'exercice ne sera pas noté, ni pris en compte pour les moyennes. Les Mag 3, en revanche n'ont plus rien à craindre : avec leur diplôme presque assuré, ce qui les préoccupe est la vie active qui arrive à grands pas.

41 Certaines études (Annoot \& Bertin, 1997; Bertin, 1998) laissent présager que les apprenants les plus faibles sont ceux qui bénéficient le plus des enseignements multimédia. La meilleure efficacité du multimédia chez les Mag 1, dont le niveau d'anglais est plutôt scolaire et certainement plus faible que chez les Mag 3, fraîchement rentrés d'un programme d'échange dans une université anglophone, reposerait, éventuellement, sur ce phénomène. Or d'après les résultats d'autres études (Cazade, 2004), ce sont les apprenants les plus avancés qui, hors environnements didactisés, ont pu le mieux profiter de l'outil multimédia et qui ont progressé à un rythme supérieur à celui des moins avancés.

Il serait cependant difficile d'inscrire la présente étude dans une logique d'« enseignement » ou d'« apprentissage » multimédia, car il s'agit d'une série de séances réalisées dans les conditions d'examen uniquement dans le but de tester l'efficacité d'une expérience ponctuelle de lecture intensive dans les deux situations. Ce type d'évaluation a été jugé pertinent car la tâche expérimentée correspond à une activité souvent proposée dans les cours de langue du secteur LANSAD à l'université. Observons que l'enseignant s'est efforcé de rester très discret pendant les périodes de lecture et de rédaction, jouant ainsi un rôle d'examinateur plutôt que d'enseignant, mais il est bien sûr possible que son enthousiasme pour l'expérience ait varié d'une séance à l'autre et que sa manière de donner les consignes ait changé au cours d'une série de huit séances. De telles modifications de comportements verbaux et/ou non-verbaux peuvent avoir un impact significatif sur les performances et réactions des apprenants, mais nous n'avons pas essayé de contrôler ce facteur. 
43 À cet égard, il est intéressant de voir que dans une étude très récente, Tissiot (2003) a trouvé des résultats très similaires chez deux groupes d'étudiants, le premier assistant à des cours en mode multimédia, le second suivant le même enseignement présenté de manière plus traditionnelle. Les étudiants ont été affectés soit au groupe multimédia, soit au groupe classique de manière complètement aléatoire. Le niveau en anglais L2 est le même dans les deux sections, c'est-à-dire plutôt faible ou moyen-faible. Plus important, et à la différence de notre étude, ces deux groupes d'étudiants en sont au même point dans leur parcours universitaire. Les facteurs variables d'ordre psychologique auxquels nous avons fait allusion plus haut ne seraient pas intervenus dans cette étude.

\section{Conclusion}

Entre les performances des deux groupes d'apprenants, on ne constate qu'une légère différence dans les scores, ces résultats n'étant pas soumis à une analyse statistique et sans doute présentant des différences statistiquement non-significatives. Si, en effet, le groupe ayant accès aux outils multimédia a obtenu, globalement, des scores meilleurs sur une série de cinq tests, le groupe privé de ces moyens a aussi très bien fonctionné. Devant ces données, nous sommes tentée d'avancer l'hypothèse que les deux professeurs concernés ont su, chacun de leurcôté, mettre en valeur les outils dont ils ont disposé pour cet enseignement. Au vu de ces résultats et de ceux venant de notre étude, il est clair que la seule utilisation des outils multimédia ne suffit tout simplement pas à donner de l'élan aux apprenants LANSAD et que la présence d'un enseignant, dont l'action saura être savamment dosée, semble devoir jouer encore aujourd'hui un rôle décisif en matière d'efficacité didactique dans un environnement d'apprentissage des langues aidé par des supports technologiques.

Je remercie vivement Catherine Pardoux, maître de conférences en statistiques à l'Université de Paris-Dauphine d'avoir réalisé les analyses statistiques présentées dans cet article.

\section{BIBLIOGRAPHIE}

Annoot, E. et J.-C. Bertin. 1997. «Intégration des nouvelles technologies dans les formations en langue de spécialité ». Rapport du CIRTAI, Université du Havre, mai.

Bertin, J.-C. 1998 « Conception de leçons multimédia : liberté ou guidage ? ». ASp19-22, 313-331.

Cazade, A. 2000. Document de synthèse non publié pour la soutenance d'une Habilitation à diriger des recherches, Université de Poitiers, accessible sur à l'adresse <ftp://

ftp.cip.dauphine.fr/hys/Articles/HDR_Synthese.doc>.

Cazade, A. 2004. « L'écran multimédia : obstacle ou atout pour l'apprenant en langues?». ASp 41-42, 175-195. 
Tisiot, B. 2003 . « Un cours multimédia : travail comparatif avec un cours en environnement classique ». Mémoire de recherche, sous la direction de Tony Toma, et Christine Vaillant, D.E.A. de Langue anglaise de spécialités scientifiques et techniques, Université de Bordeaux 2.

Vaillant, C. 1996. «L'image : agent ou obstacle dans l'entraînement à la compréhension orale en langue étrangère ?». Séminaire au Laboratoire de Recherche (GRAMAL) des Musées de France, Louvre, 9 janvier 1996.

\section{NOTES}

1. Douze étudiants de Magistère première année ont participé à la première séance ; onze à la seconde. Les Magistère troisième année étaient au nombre de dix pour la première séance et de douze pour la seconde.

\section{RÉSUMÉS}

L'étude présentée dans l'article tente d'expérimenter l'efficacité d'une lecture intensive classique sur papier par rapport à une « lecture » ou présentation multimédia. Deux critères ont été pris en compte; l'acquisition et le réemploi du vocabulaire des modules étudiés et le nombre d'informations retenues et réutilisées par les participants. Dans une étude liminaire, avec un échantillon très limité, nous avons pu déceler un meilleur rendement à l'issue des séances de lecture traditionnelle. Lors d'une deuxième étude approfondie, deux types d'apprenants semblent se profiler : un groupe moyen-faible dont les meilleures performances ont été réalisées deux fois sur quatre en mode multimédia, et un deuxième groupe, plus avancé, dont les préférences sont moins nettes.

The article presents a two-phase study designed to test the relative merits of multimedia presentations versus traditional intensive reading exercises, with reference to performance based on two criteria: vocabulary acquisition and information retrieval and reuse. In the preliminary phase of the study, based on a small sample, learners seemed to perform better on traditional reading tasks. In a larger subsequent study, two distinct types of learners emerged: an intermediate-level group whose performance was better in the multimedia mode in two categories out of four and a more advanced and slightly older group whose preferences were less pronounced.

\section{INDEX}

Mots-clés : acquisition lexicale, lecture intensive, lecture multimédia, lecture traditionnelle, mode de lecture, multimédia

Keywords : intensive reading, multimedia, multimedia reading, reading mode, traditional reading, vocabulary acquisition 


\section{AUTEUR}

\section{EVELYN PERRY}

Evelyn Perry est maître de conférences à l'Université de Dauphine Paris 9. Elle est l'auteur de nombreux articles publiés dans ASp et Cahiers de l'APLIUT. Ses recherches sont axées sur

l'acquisition du vocabulaire, ainsi que sur l'analyse syntaxique de la production écrite en anglais L2, avec une attention toute particulière portée sur le groupe nominal. Depuis quatre ans, dans le cadre du CICLaS à Paris-Dauphine et en partenariat avec l'American University (Washington, D.C.), elle dirige un projet de recherche dont l'un des objectifs est de constituer un corpus d'anglais économique, composé pour partie de textes fournis par des étudiants outre-Atlantique en économie appliquée. Elle participe à un groupe d'étude sur le suivi apprenant au sein de RANACLES. 